\title{
First principles modeling of exciton-polaritons in polydiacetylene
}

\section{chains}

\author{
Antonios M. Alvertis ${ }^{1, *}$, Raj Pandya ${ }^{1}$, Claudio Quarti ${ }^{2,3}$, Laurent Legrand ${ }^{4}$, Thierry Barisien ${ }^{4}$, Bartomeu \\ Monserrat $^{5}$, Andrew J. Musser ${ }^{6}$, Akshay Rao ${ }^{1}$, Alex W. Chin ${ }^{4}$, David Beljonne ${ }^{2, \dagger}$ \\ ${ }^{1}$ Cavendish Laboratory, University of Cambridge, J. J. Thomson Avenue, Cambridge CB3 0HE, United Kingdom \\ ${ }^{2}$ Laboratory for Chemistry of Novel Materials, University of Mons, Place du Parc, 20, B-7000 Mons, Belgium. \\ ${ }^{3}$ Université de Rennes, ENSCR, INSA Rennes, CNRS, ISCR (Institut des Sciences Chimiques de Rennes) - UMR 6226, F-35000 \\ Rennes, France \\ ${ }^{4}$ Sorbonne Université, CNRS-UMR 7588, Institut des NanoSciences de Paris, INSP, 4 place Jussieu, F-75005, Paris, France. \\ ${ }^{5}$ Department of Materials Science and Metallurgy,University of Cambridge, 27 Charles Babbage Road, Cambridge CB3 0FS, U.K. \\ ${ }^{6}$ Department of Chemistry and Chemical Biology, Cornell University, Ithaca, NY 14853 \\ *e-mail: ama80@cam.ac.uk

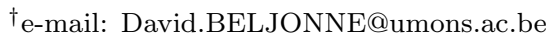

June 23, 2020

\begin{abstract}
Exciton-polaritons in organic materials are hybrid states that result from the strong interaction of photons and the bound excitons that these materials host. Organic polaritons hold great interest for optoelectronic applications, however progress towards this end has been impeded by the lack of a first principles approach that quantifies light-matter interactions in these systems, and which would allow the formulation of molecular design rules. Here we develop such a first principles approach, quantifying light-matter interactions. We exemplify our approach by studying variants of the conjugated polymer polydiacetylene, and we show that a large polymer conjugation length is critical towards strong exciton-photon coupling, hence underlying the importance of pure structures without static disorder. By comparing to our experimental reflectivity measurements, we show that the coupling of excitons to vibrations, manifested by phonon side bands in the absorption, has a strong impact on the magnitude of light-matter coupling over a range of frequencies. Our approach opens the way towards a deeper understanding of polaritons in organic materials, and we highlight that a quantitatively accurate calculation of the exciton-photon interaction would require accounting for all sources of disorder self-consistently.
\end{abstract}




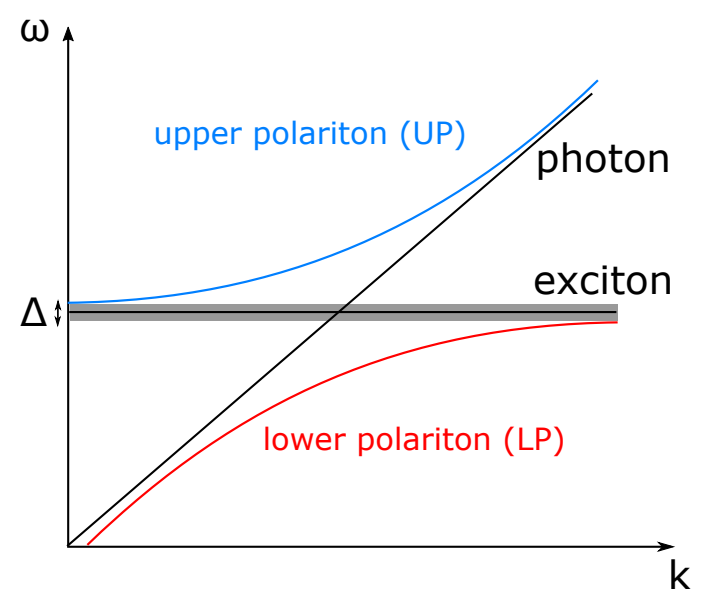

Figure 1 The dispersion of a photon in a material intersects with that of an exciton, and their interaction leads to two new hybrid polariton states. The upper and lower polaritons are separated by an energy gap of width $\Delta$ (shaded region), which is referred to as the stop-band.

\section{Introduction}

Excitons are bound electron-hole pairs that appear in semiconductors, and are critical to the optoelectronic response of these materials. These quasiparticles are connected to a finite oscillating polarization that emits an electromagnetic wave, which can act on an incident wave, leading to a light-matter interplay. Once a photon is close to resonance with an exciton, light-matter coupling can become strong, and the two cannot be treated independently, as it was first realized in the pioneering works of Pekar ${ }^{1 / 2}$ and Hopfield ${ }^{3}$ more than six decades ago. This coupling between polarization and photons is captured by introducing the quanta of a new quasiparticle referred to as a polariton, which describes the propagation of electromagnetic energy in matter. ${ }^{4}$

Organic semiconductors are a class of materials the light-matter interactions of which are relevant to a number of promising optoelectronic applications, making the understanding of their polariton properties interesting from a technological point of view. Their applications include light-emitting diodes (LEDs),$\frac{5}{5}$ solar cells ${ }^{6}$ and room temperature polariton lasers $\frac{7] 8}{8}$ Excitons in organic semiconductors are typically strongly bound $\sqrt{9}[10$ Frenkellike excitons, $\frac{11}{14}$ and their dispersion can be considered to be approximately flat ${ }^{15}$ compared to the strong dispersion of light, as visualized in Figure1. For an incident photon close to resonance with an exciton state of an organic semiconductor, strong coupling leads to two new polariton states that are referred to as upper polariton (UP) and lower polariton (LP) respectively. As depicted in Figure1, this results in a stop-band (shaded region), where no light propagation takes place within the material. The width $\Delta$ of the stop-band depends on the magnitude of the exciton-photon interaction. The stop-band is also related to, but is distinct from, the so-called Rabi splitting that is commonly used to quantify light-matter interactions, and which is defined as the maximal vertical distance between the upper and lower polariton branches!16 Polaritons in organic semiconductors have often been studied for molecular systems placed between highly reflecting mirrors that confine incident light $8[17[18$ This microcavity effect leads to a standing electromagnetic wave that interacts with the material system. However, here we focus on the case of unconfined photons, and not on such microcavity polaritons.

The building block of organic semiconductors is often organic molecules, the large size and weak intermolec- 
ular van der Waals interactions of which lead to phonon modes with low frequencies, hence strongly activated at room temperature. These slow nuclear motions lead to large variations of the electronic interactions between neighboring molecules $\frac{19 \mid 20}{20}$ Since excitons can spatially extend over several molecules, $21 \mid 22$ such low-energy phonons can have a strong influence on their properties. ${ }^{23}$ Similarly, organic semiconductors based on conjugated polymers exhibit very low-frequency phonon modes that are strongly coupled to their excitons. ${ }^{24}$ However, it is not only vibrations of low energies that couple strongly to excitons, as in organic systems high-frequency motions such as carbon-carbon stretches have often been discussed to dominate this interaction $25 \sqrt[26]{26}$ This strong coupling of excitons to the vibrations of organic semiconductors is expected to also have an impact on exciton-photon coupling, and indeed it has been shown that vibrations have a strong effect on the dynamics of polariton states $[27+31$

Therefore, understanding the properties of polaritons requires accounting not only for exciton-photon, but also exciton-vibration interactions, through a Hamiltonian operator of the form:

$$
H=H_{\text {exc-ph }}+H_{\text {exc-vib }}
$$

where the operator $H_{\text {exc-vib }}$ describes the exciton-vibration interactions. For a single exciton state with energy $\omega_{\text {exc }}$, coupled to a photon of energy $\omega_{\mathrm{ph}}$, the exciton-photon Hamiltonian can be written within the so-called resonance approximation! 32

$$
H_{\text {exc-ph }}=\omega_{\mathrm{ph}} \hat{a}^{\dagger} \hat{a}+\omega_{\mathrm{exc}} \hat{c}^{\dagger} \hat{c}-i g\left(\hat{a} \hat{c}^{\dagger}-\hat{a}^{\dagger} c\right)
$$

Here $a^{\dagger}(a)$ is the creation (annihilation) operator of the photon state, and $c^{\dagger}(c)$ the creation (annihilation) operator of the exciton. The constant $g$ quantifies the magnitude of the exciton-photon interaction and is related to the width of the stop-band $\Delta$ of Figure 1 via the relationship: $g=\frac{1}{4} \sqrt{\omega_{\mathrm{ph}} \Delta}$.

While first principles approaches have been used in several cases to parametrize the exciton-vibration interactions encoded in $H_{\text {exc-vib }} \stackrel{33] 34}{34}$ the same cannot be said for the exciton-photon Hamiltonian of equation 2. To the best of our knowledge, a comprehensive ab initio method for modeling the stop-band of organic systems is thus far missing. Naturally, experiment can inform computational models on the range of values of $\Delta$, however this does not allow for a truly predictive approach of modeling light-matter interactions in these systems. It also remains unclear how vibrational effects affect the stop-band.

Here we develop a theoretical framework for simulating exciton-photon interactions in organic systems fully from first principles. We exploit the relationship of the microscopic relation equation 2 to the classical macroscopic theory of polaritons to provide a first principles extraction of the stop-band width $\Delta$. We exemplify our approach by studying variants of the conjugated polymer polydiacetylene (PDA) (Figure2), and we show that the conjugation length of the polymer chains has a large impact on the magnitude of exciton-photon interactions. Exciton properties of PDA are obtained from first principles by using accurate Green's function based methods ( $G W$-BSE) $\stackrel{35}{37}$ which are known to be appropriate for the study of conjugated polymers, $\frac{38}{3}$ due to successfully capturing the large intrinsic correlation length of the electrons and holes that participate in exciton 
states. We compare the simulated results to experimental reflectivity measurements of the stop-band width, and we find the two to be in reasonable agreement. However, by only considering the effect of the main exciton peak on incident photons, the internal structure of the stop-band as it appears in reflectivity measurements is not reproduced in the calculation. By coupling $G W$-BSE calculations for the excitons, to non-perturbative finite differences methods for the vibrations 3904 we show that phonon effects have an important effect on the internal stop-band structure.

The structure of this paper is as follows. Section 2 provides an overview of our theoretical approach for describing light-matter interactions. We apply our methodology to disorder-free PDA in section 3 . An overview of the studied systems is also provided in this section, alongside a discussion of their exciton properties and the experimental determination of the magnitude of light-matter interactions. We then proceed to discuss the role of static disorder in the sense of limiting the infinite conjugation length of disorder-free chains, in section 4 The impact of dynamic disorder (vibrations) on light-matter interactions is discussed in section 5 , along with a detailed outline of the employed methodology for accounting for its effect. We summarize the entirety of our results and discuss potential future avenues in the Conclusions of section 6 .

\section{Theoretical background}

Consider an electromagnetic wave $\mathbf{E}, \mathbf{B}$ interacting with a material of dielectric $\epsilon(\omega)$. The wave equation for the electric field in frequency space reads:

$$
\nabla^{2} \mathbf{E}(\mathbf{r}, \omega)+\frac{\omega^{2}}{c^{2}} \epsilon(\omega) \mathbf{E}(\mathbf{r}, \omega)=0
$$

For the moment, and for the sake of simplicity, let us consider a bulk isotropic material, for which the electric field can be treated as a plane wave:

$$
\mathbf{E}(\mathbf{r}, \omega)=E \cdot e^{i(\mathbf{k} \cdot \mathbf{r}-\omega t)}
$$

By substituting equation 4 into 3 we obtain:

$$
\left[-k^{2}+\frac{\omega^{2}}{c^{2}} \epsilon(\omega)\right] E \cdot e^{i(\mathbf{k} \cdot \mathbf{r}-\omega t)}=0
$$

The transverse eigenmodes of the system satisfy the condition $E \neq 0$, therefore:

$$
c^{2} k^{2}=\omega^{2} \epsilon(\omega)
$$

It thus becomes obvious that if one obtains the dielectric response $\epsilon(\omega)$ of a material, the dispersion of the light field in the material, i.e. the polariton dispersion, can be obtained by solving equation 6 This method is agnostic to the level at which the electronic structure is computed, and our $G W$-BSE methodology provides but one possibility. 
Let us now consider the so-called resonance approximation to equation 6, as a useful way of gaining physical intuition on the origins of the stop-band. However, we emphasize that we do not make use of this approximation to obtain the simulated results presented in this paper, and it only serves as a way of developing intuition. Unless we are at resonance with an excited state $\omega=\omega_{o}$, the imaginary part of the dielectric response is negligible, therefore equation 6 can be approximated as:

$$
c^{2} k^{2}=\omega^{2} \operatorname{Re}(\epsilon(\omega))
$$

Equation 7 shows that there will only exist real solutions for the wavevector $k$ once $\operatorname{Re}(\epsilon(\omega))$ is positive. While this is generally the case, $\operatorname{Re}(\epsilon(\omega))$ can become negative in the vicinity of an optical transition $i$ with a high oscillator strength $f_{i}$. This can be seen within a Lorentz oscillator model of the dielectric response: 41

$$
\epsilon(\omega)=\epsilon_{0}+\frac{N e^{2}}{m} \sum_{i} \frac{f_{i}}{\omega_{i}^{2}-\omega^{2}-i \omega \gamma_{i}}
$$

The real part can be written as:

$$
\operatorname{Re}(\epsilon(\omega))=\epsilon_{0}+\frac{N e^{2}}{m} \sum_{i} f_{i} \frac{\omega_{i}^{2}-\omega^{2}}{\left(\omega_{i}^{2}-\omega^{2}\right)^{2}+\omega^{2} \gamma_{i}^{2}}
$$

where $\epsilon_{0}$ is the background dielectric response, $N$ the number of molecules per unit volume, $m$ the electron mass and $\gamma_{i}$ the dephasing rate. In the vicinity of a transition $i, \omega=\omega_{i}+\delta \omega$, and only keeping terms in first order of $\delta \omega$ we obtain the condition for $\operatorname{Re}(\epsilon(\omega))<0$ to be:

$$
f_{i}>\left(1+\frac{\omega_{i}}{2 \delta \omega}\right) \cdot \frac{\gamma_{i}^{2}}{\omega_{p}^{2}}
$$

where $\omega_{p}^{2}=\frac{N e^{2}}{\epsilon_{0} m}$ the plasma frequency of a material. The width of the frequency region over which condition 10 holds and thus $\operatorname{Re}(\epsilon(\omega))<0$, defines the stop-band width $\Delta$.

Therefore, the brighter an optical transition, the more likely it is that it will lead to a forbidden region for light propagation in the material. Since the oscillator strength of a material satisfies the sum rule:

$$
\sum_{i} f_{i}=Z
$$

with $Z$ the number of electrons per molecule, we conclude that materials with fewer bright optical transitions are more likely to satisfy condition 10 and lead to strong light-matter interactions. This provides the motivation for studying extended one-dimensional systems like PDA, as elaborated in the following section.

Before proceeding to the results section, we would like to highlight two points regarding the range of applicability of the presented methodology. First of all, this has been discussed in the context of a general Lorentz oscillator, and the frequencies $\omega_{i}$ appearing in equation 8 need not correspond to exciton states. While here we focus on exciton-polaritons, optical phonons and plasmons can also form hybrid states with light, 42,43 and the presented theoretical framework could be used to describe such phenomena. Secondly, the polariton properties 


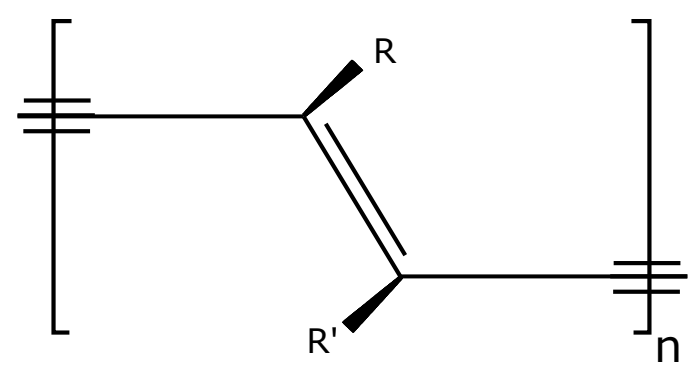

Figure 2 The structure of a diacetylene monomer, which generally coincides with the unit cell of an infinite and periodic crystal of polydiacetylene.

we calculate correspond to the bulk of an infinite material structure. However, these states can also form at the surface of a material, only propagating along the interface between different media. These states are referred to as surface polaritons, and have a finite dispersion within the stop-band of the infinite 'bulk' polariton states that we study here $\stackrel{44}{4}$ Our theoretical framework does not capture such surface effects, but could be extended in that direction in the future.

\section{Application to disorder-free PDA}

Polydiacetylene (PDA) is a conjugated polymer with an alternating sequence of single-double-single-triple carbon-carbon bonds, as visualized in Figure2. These materials are known to give very high-quality crystals, with an almost perfect alignment of the polymer chains parallel to each other and free from conformational defects, due to the topochemical reaction they originate from. $\stackrel{45}{ }$ Variants of PDA are traditionally named after the color of the transmitted light through a film of the material. Here we study two PDA variants shown in Figure 3 alongside their absorption and reflection spectra. We refer to the structure of Figure 3 a that absorbs at lower energies as 'blue' $\mathrm{PDA} 4 \sqrt{46}$ and the one of Figure $3 \mathrm{~b}$ that absorbs at higher energies as 'red' 47448 It is important to note that the two types of PDA chains and their optical responses should in no way be associated to varying levels of disorder in their structures but are largely determined by their backbone geometries (see below) $\underline{49}$

We determine the stop-band of the two PDA variants by measuring their reflectivity spectra presented in Figure3, for near-normal incidence of light. Details of the experimental measurements are given in Appendix A. If we were to disregard the absorption from the exciton over a finite region of frequencies, then every incident photon with an energy within the stop-band region would be reflected, giving a reflectance of $R=1, \sqrt[50]{ }$ since no propagation of light can take place within the forbidden region. However, the finite damping $\gamma$ that appears in the dielectric function of equation 8 due to the absorption of an exciton, leads to weaker reflectance and a 'gap' within the forbidden region of the stop-band $4 \sqrt[41]{51}$ (as also seen in Figure $7 \mathrm{~b}$ for a model PDA system). Further gaps to the high reflectivity of the stop-band can appear due to disorder or additional bright exciton peaks. Therefore, it is not always the case that one can identify the stop-band as a region of high reflectance, and its internal structure can be rich. This is the case with the reflectivity spectra of Figure 3 . Nevertheless, we can still identify the width of the stop-band as the distance of two peaks in the reflectivity spectra: the one which 

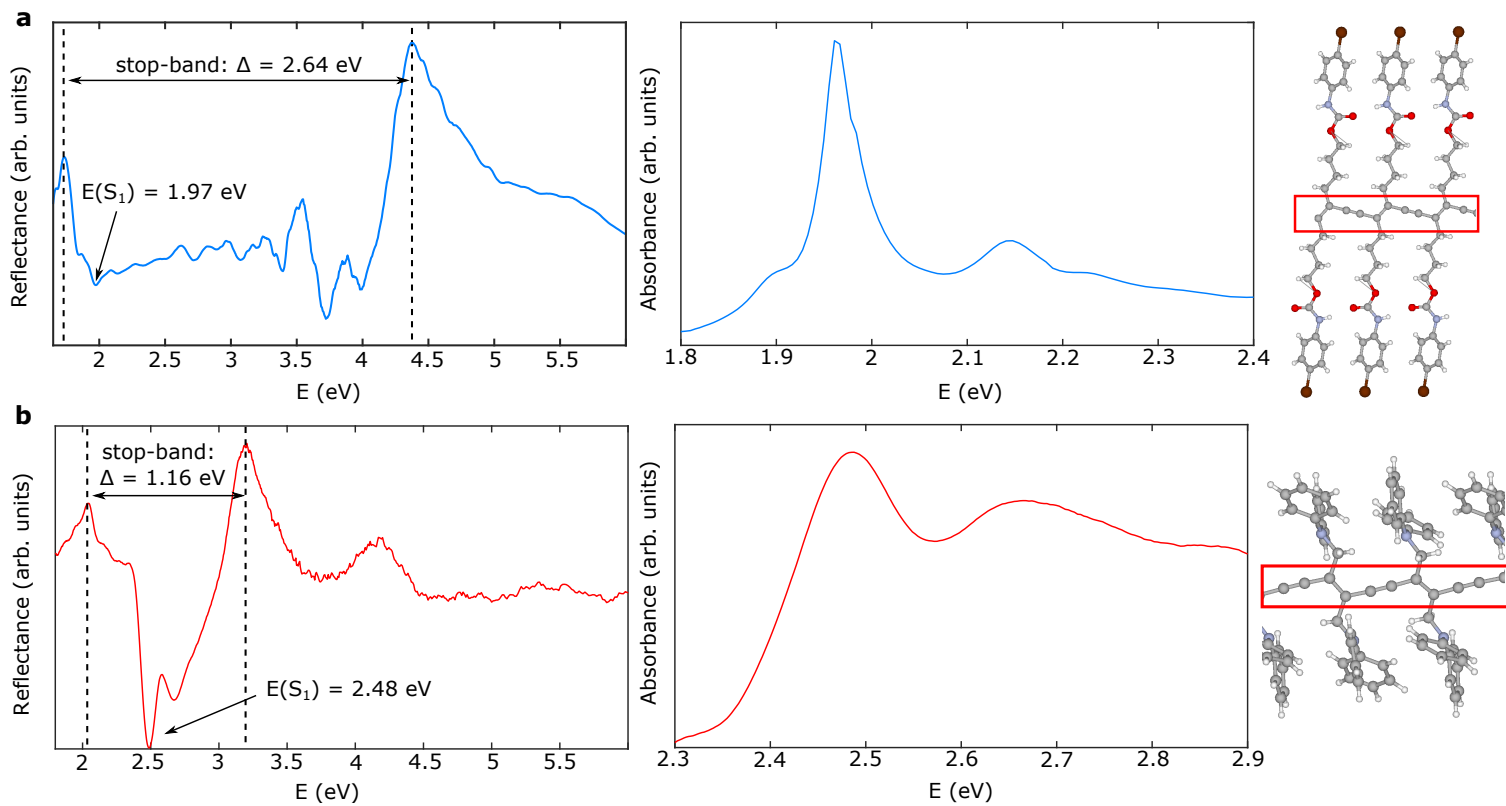

Figure 3 Experimental reflection and absorption spectra of two studied variants of polydiacetylene: $\mathbf{a}$ blue chains and $\mathbf{b}$ red chains. The stop-band width of the two structures is approximately determined from the reflectivity spectra. The polymer structures are given alongside the experimental plots, with the polymer backbone highlighted in red rectangles, in order to distinguish it from the side-chains. Carbon atoms are denoted in light gray, hydrogen in white, oxygen in red, nitrogen in light blue and bromine in brown.

appears on the low-energy side of the dip due to the absorption from an exciton state, and the one for which the reflectance reaches values at least as large as the maximum of the first peak. This way of determining the stop-band is similar to previous work,$\left[51\right.$ and strong light-matter coupling in squarylium ${ }^{52}$ provides a particularly good example of using the reflectivity measurements to that end.

Our reflectivity measurements show that the stop-band width is $2.64 \mathrm{eV}$ and $1.16 \mathrm{eV}$ for the blue and red chains respectively. We discuss the origin of this large difference between the red and blue chains with the aid of simulations below. The measured values are within the expected range, as the stop-band width has been shown to surpass values of $1-2 \mathrm{eV}$ in several organic materials. $\frac{51}{1 n}$ particular, it has been shown that for near-normal incidence of light on a lattice of aligned dipoles, values of this order of magnitude might be obtained!51 Given the close-to-perfect alignment of the polymer chains in PDA, as well as the large oscillator strength of their lowest bright exciton state, it is not surprising that light-matter coupling is strong.

Let us now examine some of the details of the experimental reflectivity spectra. For the blue chains, the reflectance dips around the energy of the bright singlet exciton state at $1.97 \mathrm{eV}$, and a vibronic peak is also weakly resolved at $2.15 \mathrm{eV}$. Furthermore, a small dip appears at $1.85 \mathrm{eV}$, and this has been discussed to be due to minority chain populations with different geometries, which are present within the samples $\underline{49}$ Additional structure appears at energies of $2.72 \mathrm{eV}$ and $3.06 \mathrm{eV}$. It is currently not clear what these peaks correspond to, however the side-chains that are connected to the conjugated PDA structure can host exciton states of their own, potentially leading to some of the observed features. At higher energies, the reflectance shows an increase at approximately $3.5 \mathrm{eV}$ and then decreases again. This is due to exciton states of unpolymerized diacetylene monomers which are known to appear in this energy range ${ }^{49}$ Finally, there is a stark increase of 


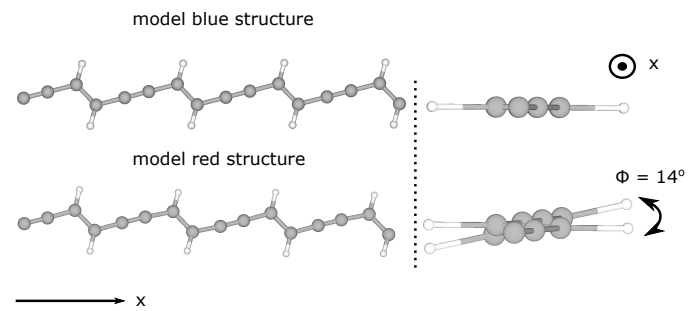

Figure 4 Model PDA structures, constructed by substituting the side-chains of the real materials of Figure 3 with hydrogen atoms. While the two model systems might look identical when viewed from the side (left), viewing them in the direction in which the chains lie (right) emphasizes that the blue structure is planar, while a finite torsion occurs between subsequent monomers of the red structure.

the reflectance as photon energies approach $4.4 \mathrm{eV}$, which is then followed by a continuous drop in its value, behavior characteristic of the stop-band edge. For the red PDA, a large dip in the reflectance appears at $2.48 \mathrm{eV}$ where the bright exciton state of this structure absorbs, and a vibronic peak is then present at $2.68 \mathrm{eV}$. The reflectance then increases steadily until reaching the edge of the stop-band. On the low-energy side of the main exciton peak, some additional structure is present, and similar to the case of blue PDA, we attribute this to minority chain populations.

The PDA variants of Figure 3 contain a very large number of atoms in their unit cells, due to the size of the side-chains connected to the conjugated structure (highlighted with a red rectangle in Figure 3). In order to make the computational problem of obtaining accurate exciton properties for PDA feasible, we resort to studying two simplified model PDA chains, where the side-chains have been substituted by hydrogen atoms, as visualized in Figure4. The lattice parameters, as well as the interatomic distances within the conjugated chains for the two model PDAs, are kept to be the same as for the respective real structures. When viewing the model PDA chains sideways as on the left of Figure 4 these systems look identical. However, by looking into the direction along which the chains extend, it becomes clear that the model red structure is not planar, and there exists a finite torsional angle of $14^{\circ}$ between subsequent diacetylene monomers. The effect of this torsion has been discussed in detail previously, $[53$ and shown to lead to significant differences in the optoelectronic response of the two systems. In crystalline PDAs the side groups, through their mutual organization, pilot the chain conformation that in turn governs the electronic properties. However, the side groups themselves do not participate to the conjugated system, justifying the substitution by hydrogen atoms. Nevertheless, this simplification leads to a renormalization of exciton energies. This is due to the the strong dielectric mismatch introduced between the conjugated chain and the outside medium, as discussed below.

The absorption of the model PDAs and real red system as computed from $G W$-BSE is given in Figure 5 . The computational details for the electronic structure calculations are given in Appendix B. We were not able to obtain converged exciton properties for the real blue PDA system, and we exclude it from any further computational analysis. The first thing to observe from Figure 5 is that there is only a single bright transition for all the studied systems, at least in the idealized case of the infinite periodic material. Therefore, PDA provides an ideal system for satisfying condition 10 and observing strong light-matter interactions, since the oscillator strength is not shared between several transitions according to the sum rule of equation 11 . It should however be 


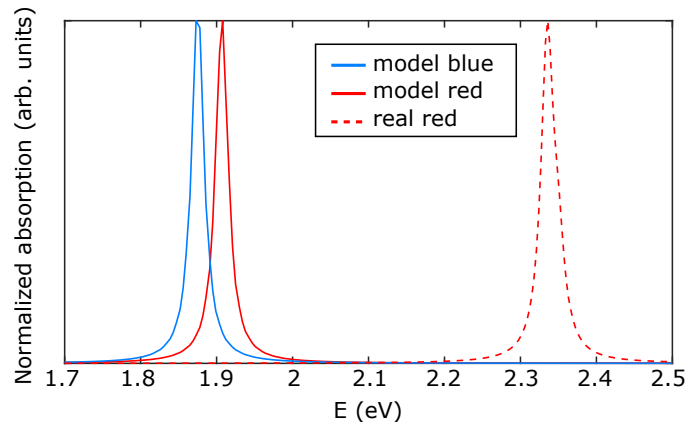

Figure 5 Normalized absorption of the two model PDA chains and the real red structure.

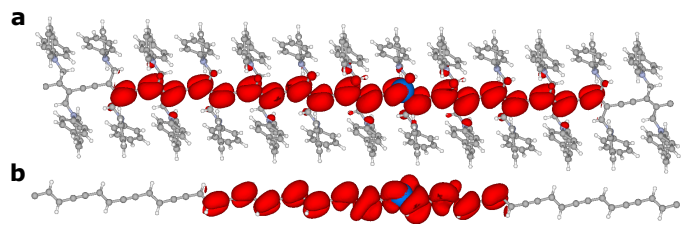

Figure 6 Exciton wavefunction of $\mathbf{a}$ the real red PDA chains and $\mathbf{b}$ the model red chains. The electron density is visualized in red for a hole fixed at the position indicated by a blue sphere.

noted that the real systems has some additional exciton states with small but non-vanishing oscillator strength at energies above $3 \mathrm{eV}$, which are associated with the side-chains.

Moreover, it is interesting to note the difference in the exciton energy between the real and model red structures. Since the polymer backbone is identical in both cases, it is only the side-chains that can be causing the observed difference. The side-chains of the real red PDA consist of two phenyl rings, which are highly polarizable and hence provide significant screening to the Coulomb interaction that binds electron-hole pairs together. In contrast, in the model system the field lines of this Coulomb interaction are not screened by a medium, since the side-chains have been substituted by hydrogen atoms. Consequently, the exciton is more strongly bound in the model chain, further stabilizing its energy. For the real blue system, the side-groups largely consist of non-polarizable alkyl chains, hence the screening they provide is much weaker compared to the red PDA. This is reflected by the fact that the exciton energy of the model blue system, where the side-chains have been substituted by hydrogen atoms, closely reproduces the experimental value from the absorption spectrum of Figure $3 \mathrm{a}$. Table 1 summarizes the values for the fundamental gaps of the chains, along with the energy of the excitons and their binding energy. The exciton binding energy of the real red system is equal to $0.52 \mathrm{eV}$, while for both model chains it is greater than $2 \mathrm{eV}$, emphasizing the large impact of the screening provided by the polarizable side-chains. This effect can be further emphasized by visualizing the exciton wavefunctions of the real and model red chains in Figure6. The electron density is visualized in red, for a hole fixed at the position indicated by a blue sphere. As expected, the lack of side-chain screening of the Coulomb interaction between the electron and hole in the case of the model chain leads to an exciton that is spatially more confined than in the real system. As far as the relative electron-hole motion is concerned, we also reach a good agreement with the results from electroabsorption studies that provide an exciton bohr radius of several nanometers, corresponding to an exciton covering a few monomer units in the blue and red conformations $\underline{48 \mid 54}$ 
Having obtained $\epsilon(\omega)$ from $G W$-BSE electronic structure calculations of the three materials, we proceed to obtain the polariton dispersion. Due to the almost perfect alignment of PDA chains along one axis $(x)$, we use the polariton dispersion relation! $\sqrt{51}$

$$
c^{2} k^{2}=\omega^{2} \epsilon_{x x}(\omega),
$$

Here $\epsilon_{x x}(\omega)$ is the only non-zero component of the dielectric tensor of the material, and in the $y$ and $z$ directions, the vacuum dispersion holds: $\omega^{2}=k^{2}$. We obtain the component $\epsilon_{x x}(\omega)$ of the dielectric tensor, by solving the Bethe-Salpeter equation for incident light normal to the chains, which approximately emulates the experimental conditions used to obtain the reflectivity spectra of Figure 3 . Figure 7 a visualizes the computed dispersion for the example case of the model blue chains, highlighting the resulting stop-band of $2.23 \mathrm{eV}$. The $\Delta$ values obtained for all studied systems are are summarized in Table2, and the experimental values are given for comparison. It should be emphasized that even when we include the effect of exciton dispersion on the polariton states, the resulting theory values for the stop-band width remain largely unchanged. We see from Table 2 that the model blue chains have significantly larger stop-band width than the model red system. Since the only significant difference between these structures is the torsional angle between subsequent diacetylene monomers (see Figure 4), we conclude that the magnitude of this torsion has a strong effect on the magnitude of lightmatter interactions. Indeed the finite torsion of the model red system leads to deviations from the perfect dipole alignment of model blue PDA, and which is crucial to facilitating strong coupling to photons. The computed values for the stop-band width are in reasonable agreement with the experimental values of Figure 3 , and the large difference between the blue and red structures is captured.

Furthermore, the comparison of the predicted $\Delta$ values for the real and model red systems suggests that the introduction of polarizable side-chains leads to weaker exciton-photon interactions. This is likely due to two reasons. Firstly, the dielectric screening that they provide to electron-hole pairs (Figure6) leads to a more weakly bound exciton, which has a higher transition energy compared to the model system. Hence the condition of equation 10 becomes more difficult to satisfy. This argument only holds for polarizable side-chains that provide significant screening, which is not the case for the real blue PDA, justifying the use of the model blue system to approximate its stop-band in Table2 2 Moreover, upon the introduction of side-groups, a few exciton states with small but non-vanishing oscillator strength appear at energies above $3 \mathrm{eV}$, hence reducing the brightness of the main exciton peak according to the sum rule of equation 11, and also making the frequency range that satisfies the condition of equation 10 narrower.

The values predicted by the calculations for the stop-band width are in reasonable agreement with experiment, as summarized in Table2. We believe that the differences are largely due to our modeling of a perfect periodic structure and ignoring exciton resonances which are present in a realistic film, for example due to unpolymerized diacetylene monomers and minority chain populations at different geometries. Each of these weak transitions couples to photons and has a (narrow) stop-band of its own. The overlap of the different stop-bands due to the various weak exciton resonances with that of the main exciton peak leads to an overall 
wider stop-band 4 In agreement with this line of thought, the predicted values underestimate experiment for both materials.

For normal incidence of light, the reflectance can be calculated as:

$$
R(\omega)=\frac{(n(\omega)-1)^{2}}{(n(\omega)+1)^{2}}
$$

where $n(\omega)$ the complex refractive index of the material. For the model blue chains this is plotted in Figure $7 \mathrm{~b}$, where the stop-band is clearly resolved. The absorption from the exciton state results in a low-reflectance gap within the stop-band, which is in agreement with experiment. However, the computed reflectivity spectrum looks qualitatively very different from the experimental one of Figure 3 a. This is due to entirely ignoring the effects of disorder in the calculation. The presence of minority chain populations with different geometries, as well as unpolymerized diacetylene monomers are ignored, and the exciton states associated with these would lead to more gaps in the reflectivity. For the model chains we ignore excitons associated with the side-chains, which would also introduce additional structure within the stop-band. We will see later that dynamic disorder is another important factor towards determining the internal structure of the stop-band.

Before moving on to consider the effects of disorder, we would like to comment on the speed of energy transfer in these materials, as predicted by the results of this section. First, let us consider the case of purely excitonic motion. The quantity that we need to compute in order to quantify the speed of band-like exciton motion is the exciton group velocity:

$$
v_{\mathrm{g}, \mathrm{exc}}=\frac{1}{\hbar} \cdot \frac{\partial E_{\mathrm{exc}}}{\partial q}
$$

where $E_{\text {exc }}$ the exciton energy and $q$ the exciton wavenumber. Following photoexcitation, a wavepacket is generated on the exciton potential energy surface, the momentum of which is narrowly distributed around $q=0$, since light induces close-to-vertical transitions within the Franck-Condon approximation. The center of mass of this $\Gamma$-point exciton will then travel with the velocity of equation 14 We are interested in energy transfer in the direction of the chains, which we define to lie along the $\mathrm{x}$ axis, we thus approximate $v_{\mathrm{g}, \text { exc }}$ as the average along the respective dimension of reciprocal space. To this end, we calculate the exciton energy at the band-edge $X$, using a supercell calculation that is outlined in Appendix $\mathrm{B}$, and we obtain:

$$
v_{\mathrm{g}, \mathrm{exc}} \approx \frac{1}{\hbar} \cdot \frac{E_{\mathrm{exc}}(X)-E_{\mathrm{exc}}(\Gamma)}{q(X)-q(\Gamma)}=3 \cdot 10^{5} \mathrm{~m} / \mathrm{s},
$$

Now let us consider the group velocity of a polariton $v_{\mathrm{g}, \mathrm{pol}}$ close to the exciton resonance, which can be computed from the solution of equation 12 for different energies of the incident light. The result for the model blue chains is given in Figure $8 \mathrm{p}$; at low frequencies, the speed of light is $c=c_{o} / n$. However, as we approach the energy of the exciton transition, the exciton content of the lower polariton state becomes larger, reducing the speed of propagation. Figure $8 \mathrm{~b}$ provides a closer view to the region around resonance, and we find that the polariton propagates with a speed of the order of $10^{6} \mathrm{~m} / \mathrm{s}$, which is an order of magnitude faster than purely 


\begin{tabular}{cccc}
\hline \hline & $\mathrm{E}\left(\mathrm{S}_{1}\right)(\mathrm{eV})$ & $\mathrm{E}_{g}(\mathrm{eV})$ & $\mathrm{E}_{\text {binding }}(\mathrm{eV})$ \\
\hline model blue & 1.88 & 3.97 & 2.11 \\
model red & 1.91 & 4.62 & 2.71 \\
real red & 2.34 & 2.86 & 0.52 \\
\hline \hline
\end{tabular}

Table 1 Values for the first exciton energy $\mathrm{E}\left(\mathrm{S}_{1}\right)$, energy of the fundamental gap $\mathrm{E}_{g}$ and exciton binding energy $\mathrm{E}_{\mathrm{binding}}$ of the three PDA chains.

\begin{tabular}{ccc}
\hline \hline structure & $\Delta(\mathrm{eV})$ - theory & $\Delta(\mathrm{eV})$ - experiment \\
\hline blue & $2.23($ model system $)$ & 2.64 \\
red & 1.06 & 1.16 \\
model red & 1.45 & - \\
\hline \hline
\end{tabular}

Table 2 Values for the stop-band width of the three disorder-free PDA chains.

excitonic band-like propagation. One might observe that very close to the exciton resonance the group velocity obtains negative values, which is unphysical. This is due to the fact that in this narrow region, the material exhibits anomalous dispersion and the group velocity is generally not a useful concept in these cases 41

\section{Static disorder: the effect of finite chain length}

In a realistic film of PDA, the system deviates from the perfectly periodic behavior in more than one ways. As we saw when discussing the experimental reflectivity spectra, unpolymerized chains of diacetylene can be present, as well as minority populations of chains at different conformations. Imperfections in the chain polymerization can lead to breaks in the conjugation of the system, or even to the termination of the polymer structure after a finite number of monomers. Here we consider such effects limiting the chain conjugation, and we ignore atomic defects and vacancies, due to the pure character of the samples as discussed previously.

In order to understand the impact of finite conjugation lengths on the exciton and polariton properties, we study five different finite systems consisting of one to five diacetylene monomers, which we terminate on both sides with hydrogen atoms. For simplicity, we choose to terminate the chains after the occurrence of a triple bond, so that the carbon atoms at the boundaries only need to bond with a single hydrogen. As an example, Figure 9 visualizes a trimer of diacetylene.

We perform $G W$-BSE calculations on these oligomers, introducing a vacuum of $20 \AA$ between their periodic images. We thus obtain $\epsilon(\omega)$ for each of these, and similar to the previous section, compute the energy of the first singlet exciton $\mathrm{E}\left(\mathrm{S}_{1}\right)$ and the stop-band width $\Delta$, which we visualize in Figures $10 \mathrm{p}$ and $10 \mathrm{p}$ respectively. We have chosen to construct the oligomer chains so that they are planar, and hence by increasing the number of monomers, we approach the periodic limit of the model blue PDA chains of the previous section, which is also noted on Figure 10

First of all, it is clear from Figure 10 a that as we add more monomers to the chain, the exciton energy is stabilized and converges to the periodic limit. Due to the large computational cost, we were unable to add the required number $N$ of diacetylene monomers in order to reach the periodic limit, however by fitting a model of the form $\mathrm{E}\left(\mathrm{S}_{1}\right)=a \cdot N^{b}$ to the first five datapoints of Figure 10 , we estimate that the periodic limit is 

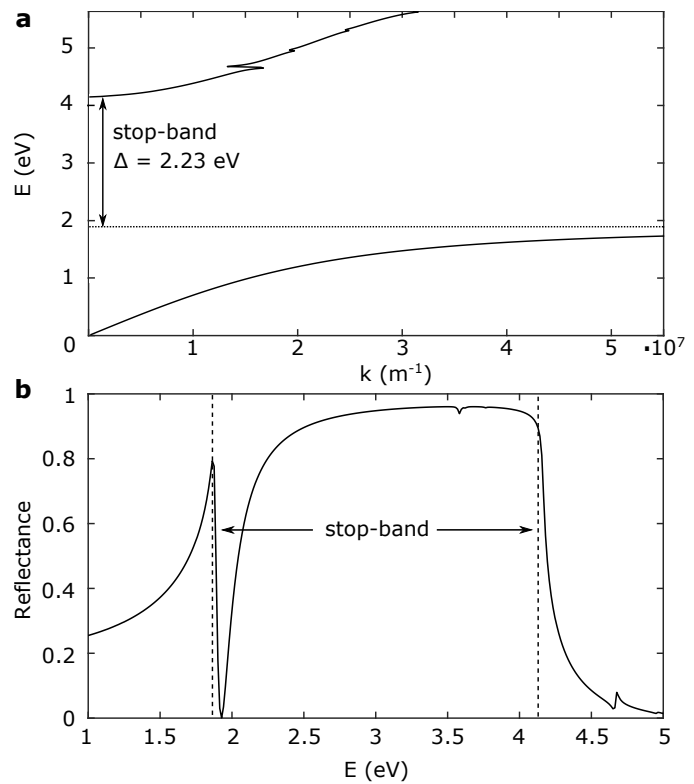

Figure 7 Calculated a polariton dispersion and $\mathbf{b}$ reflectance of the model blue PDA chains, highlighting the stop-band of width $\Delta=2.23 \mathrm{eV}$.

a

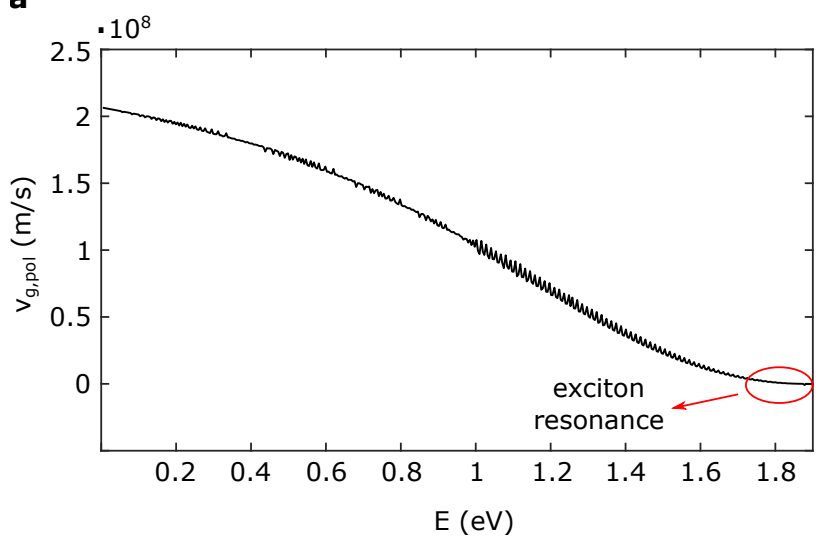

\section{b}

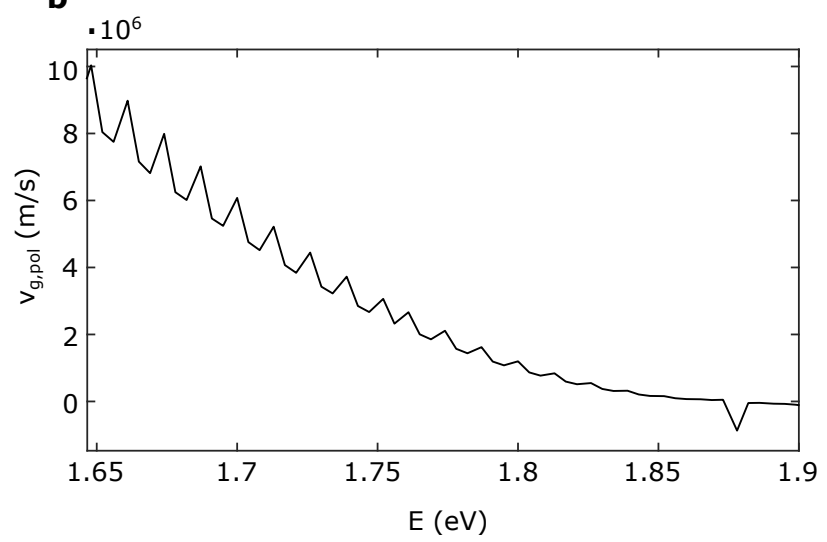

Figure 8 Polariton group velocity in model blue chains, for different energies of the incident light. Panel a shows a large region of frequencies, whereas panel $\mathbf{b}$ provides a closer view of the region close to the resonance with the exciton.

obtained for $N=15$ monomers, in very close agreement with a previous experimental study 55 Furthermore, we find that the stop-band width increases with a larger number of monomers in Figure 10p, and the periodic limit of an infinite chain provides the maximal possible magnitude for light-matter interactions. This can be rationalized from the fact that the exciton frequency appearing in the oscillator strength condition 10 decreases with increasing number of diacetylene monomers.

We therefore conclude that the presence of static disorder, in this case in the form of finite chain lengths, reduces the magnitude of light-matter interactions. However, the large measured stop-band width of $\Delta=2.64 \mathrm{eV}$ for the blue chains suggests that conjugation-limiting effects are not important in PDA. Naturally, breaks in the conjugation and imperfect polymerization do occur, however the results suggest that the vast majority of chains extend beyond 15 diacetylene monomers, for which number the periodic limit is reached, and the maximal light-matter coupling is obtained. Here we do not compute the effect of static disorder on the reflectance of 


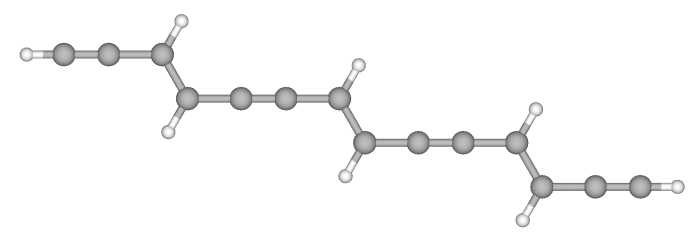

Figure 9 A hydrogen-terminated trimer of diacetylene.
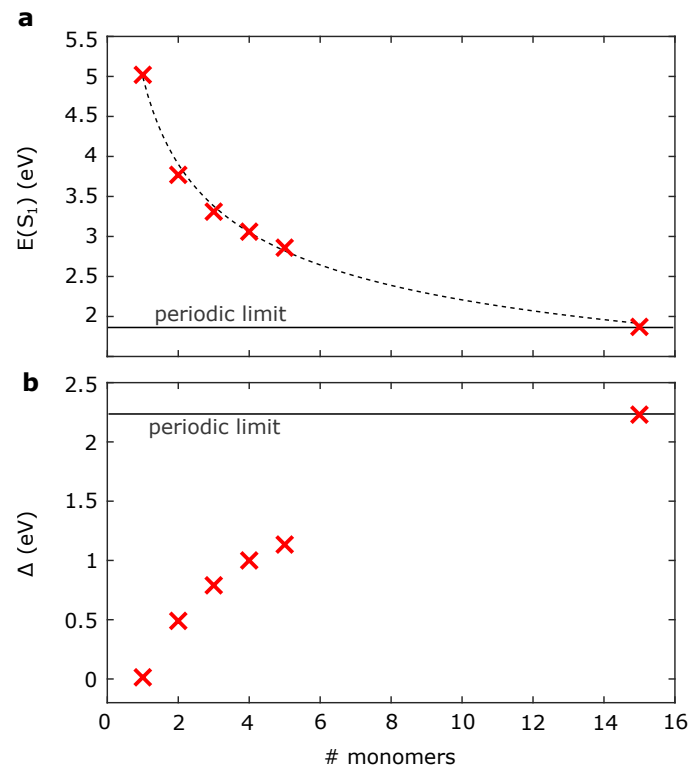

Figure 10 The exciton energy (panel a) and stop-band width (panel b) for chains consisting of a different number of diacetylene monomers $N$. Since the constructed oligomers are planar, the periodic limit of the model blue chains is approached for a larger number of monomers.

PDA, as this would require explicit inclusion of e.g. unpolymerized diacetylene monomers within a supercell of PDA, making the cost of these calculations prohibitive. We now proceed to discuss the effects of dynamic disorder on light-matter coupling.

\section{Dynamic disorder: the effect of vibrations}

A purely electronic structure calculation predicts that there is only a single bright exciton state in PDA, the vertical transition peak in the computed absorption spectrum of Figure 5 . In reality the ground- and excited-state potentials of the PDA chains are displaced along coupled vibrational normal modes, and electronic excitations involve multiple ending vibrational levels (and a thermalized distribution of starting vibrational levels). This is evident in the experimental absorption spectra of Figure 3, where one can resolve vibrationally dressed $0-1$ and $0-2$ phonon side bands on top of the purely $0-0$ electronic transition. Intuitively, it is obvious that these phonon side bands in the structure of $\operatorname{Im}(\epsilon(\omega))$, will give rise to more structure in $\operatorname{Re}(\epsilon(\omega))$ compared to the case of a single exciton peak, according to the Kramers-Kronig relation:

$$
\operatorname{Re}(\epsilon(\omega))=P \int_{0}^{+\infty} \frac{d \nu}{\pi} \operatorname{Im}(\epsilon(\nu)) \frac{2 \nu}{\nu^{2}-\omega^{2}}
$$


This effect reduces the width of the region in which $\operatorname{Re}(\epsilon(\omega))<0$ following an excitonic transition, as we show in Figure $11 \mathrm{~b}$. We call the width of this region $\Delta_{\mathrm{vib}}$, and the fact that it is smaller than $\Delta$ means that a gap in the high reflectivity of the stop-band is introduced (see Figure 11 below). We therefore proceed to study the effects of exciton-phonon coupling, as a means of understanding the internal structure of the stop-band. To this end, we employ the linear-vibronic Hamiltonian!

$$
H=\mathrm{E}\left(\mathrm{S}_{1}\right) \cdot I+\sum_{k} \hbar \omega_{k} \lambda_{k}\left(b_{k}^{\dagger}+b_{k}\right)+\sum_{k} \hbar \omega_{k} b_{k}^{\dagger} b_{k},
$$

where $I$ is the identity, $k$ runs over the phonon modes of the system, which have frequencies of $\omega_{k}$. The energy of the first singlet exciton refers to the infinite periodic limit. The Huang-Rhys factors are calculated as:

$$
\lambda_{k}=\frac{\mathrm{V}_{\mathrm{ep}, k}}{\hbar \omega_{k}}
$$

where we approximate the first derivative $\mathrm{V}_{\mathrm{ep}, k}$ of the exciton energy along mode $k$ using the finite difference formula:

$$
\mathrm{V}_{\mathrm{ep}, k}=\frac{\mathrm{E}\left(\mathrm{S}_{1}\right)_{+\delta u(k)}-\mathrm{E}\left(\mathrm{S}_{1}\right)_{-\delta u(k)}}{2 \delta u(k)},
$$

with $\delta u(k)$ being a small dimensionless displacement along mode $k$. At a practical level, the calculation of $\mathrm{V}_{\mathrm{ep}, k}$ requires two $G W$-BSE calculations at the displaced configurations of each mode, and constitutes a very computationally intensive process. We were therefore only able to obtain a full parametrization of the Hamiltonian 17 for the two model chains, which only have a small number of phonon modes due to the small size of their unit cell. The existence of the side-chains for the real red chains, for which we previously calculated the exciton properties, makes the task of computing exciton-phonon interactions a very formidable one, and we thus limit the following analysis to the model chains.

When computing the Huang-Rhys factors of PDA, one needs to account for the fact that the exciton can travel a finite distance of $N$ monomers coherently, before being scattered by a phonon. Therefore if $\lambda_{k, \text { unit }}$ is the value obtained within a unit cell exciton calculation where the phonon mode $k$ has been displaced, then the actual value of the Huang-Rhys parameter is $\underline{57}$

$$
\lambda_{k}=\frac{\lambda_{k, \text { unit }}}{N}
$$

We estimate the exciton coherence length $N$ as:

$$
N=\frac{v_{\mathrm{g}, \mathrm{exc}} \cdot \tau_{\mathrm{scatter}}}{L_{\mathrm{DA}}}
$$

where $v_{\mathrm{g}, \text { exc }}$ the exciton group velocity from section 3 , $\tau_{\text {scatter }}$ the timescale for the exciton to be scattered by a phonon, and $L_{\mathrm{DA}}$ the length of a single diacetylene monomer. Since we find that the excited state surface of the double-bond stretch is the steepest, i.e. has the largest value of $\mathrm{V}_{\mathrm{ep}, k}$, we approximate $\tau_{\text {scatter }}$ by the period of 
$23 \mathrm{fs}$ of this mode, giving us an exciton coherence length of $N=15$ monomers for both model chains, in good agreement with previous estimates on similar systems. $\frac{26}{6}$

We write the $i^{\text {th }}$ eigenstate of the Hamiltonian of equation 17 as:

$$
\left|\Psi^{(i)}\right\rangle=\sum_{k=1}^{M} \sum_{\tilde{v}}^{N_{q}} c_{\tilde{v}_{k}}^{(i)}\left|1, \tilde{v}_{k}\right\rangle
$$

where there are $M$ vibrational modes in total. The basis state $\left|1, \tilde{v}_{k}\right\rangle$ is the short-hand notation for

$$
\left|1, \tilde{v}_{k}\right\rangle=\left|1,0,0, \ldots, \tilde{v}_{k}, 0, \ldots, 0\right\rangle,
$$

i.e. the state where the exciton is populated and the first index set to one, and where among all vibrational modes, only mode $k$ is populated by $\tilde{v}_{k}$ quanta. We thus only allow for a single mode to be excited at one time, as a means of simplifying the problem. In principle, every phonon mode can admit an infinite number of quanta, however in practice we need to truncate the associated Hilbert space to only allow for up to $N_{q}$ quanta in each mode. We find that for the model PDA chains $N_{q}=3$ converges the results for the dielectric response.

Similar to previous works, 56 we include the effect of vibrations in the absorption spectrum by taking:

$$
\begin{array}{r}
\operatorname{Im}(\epsilon(\omega))=\left(c_{0}^{(1)}\right)^{2} \cdot \operatorname{Im}(\epsilon(\omega))_{0-0}+ \\
\left|\sum_{k} \sum_{\tilde{v}} c_{\tilde{v}_{k}}^{(1)} \frac{\lambda_{k}^{2 \tilde{v_{k}}} e^{-\lambda_{k}^{2}}}{\tilde{v_{k}} !}\right|^{2} W\left(\omega-\omega_{\mathrm{S}_{1}}-\omega_{k}\right),
\end{array}
$$

where $W\left(\omega-\omega_{\mathrm{S}_{1}}-\omega_{k}\right)$ a Lorentzian centered around $\omega_{\mathrm{S}_{1}}+\omega_{k}$, for which we use the same width as for the peaks of the purely electronic part $\operatorname{Im}(\epsilon(\omega))_{0-0}$ of the dielectric response, as obtained from $G W$-BSE calculations detailed in Appendix B. Moreover, in Appendix B, we show for the example case of the model blue chains, that the result for the width $\Delta_{\text {vib }}$ is largely independent of the peak width $\gamma$, for values of $\gamma$ between $5 \mathrm{meV}$ and $50 \mathrm{meV}$. The coefficients $c^{(1)}$ are obtained by diagonalizing the Hamiltonian 17 , with $c_{0}^{(1)}$ denoting the contribution from the purely excitonic basis state $|1,0,0, \ldots, 0\rangle$. Here we reside to approximating the absorbance $\alpha(\omega)$ with $\operatorname{Im}(\epsilon(\omega))$. This is a reasonable approximation for determining $\Delta_{\mathrm{vib}}$, since this quantity depends on the intensity ratio of the $0-0,0-1,0-2$ peaks etc. The absorbance $\alpha(\omega)$ and imaginary dielectric response $\operatorname{Im}(\epsilon(\omega))$ are proportional to each other, which suggests that the error we introduce is small.

For the example case of the model blue chains, we visualize the result of including phonon effects on $\operatorname{Im}(\epsilon(\omega))$ in Figure 11, while Figure 11] shows the corresponding $\operatorname{Re}(\epsilon(\omega))$ as obtained by using the Kramers-Kronig relation 16. For $\operatorname{Re}(\epsilon(\omega))$ we also draw a comparison to the case without phonons. The peaks that feature prominently in the absorption spectrum are due to the double- and triple-bond carbon stretching motions. Intuitively, one can understand why these vibrations are most strongly coupled to the exciton from the fact that they transiently lead to structures with a smaller bond-length alternation, i.e. deviating from the Peierlsdistorted structure that gives conjugated polymers such as PDA their semiconducting character, and bring the 


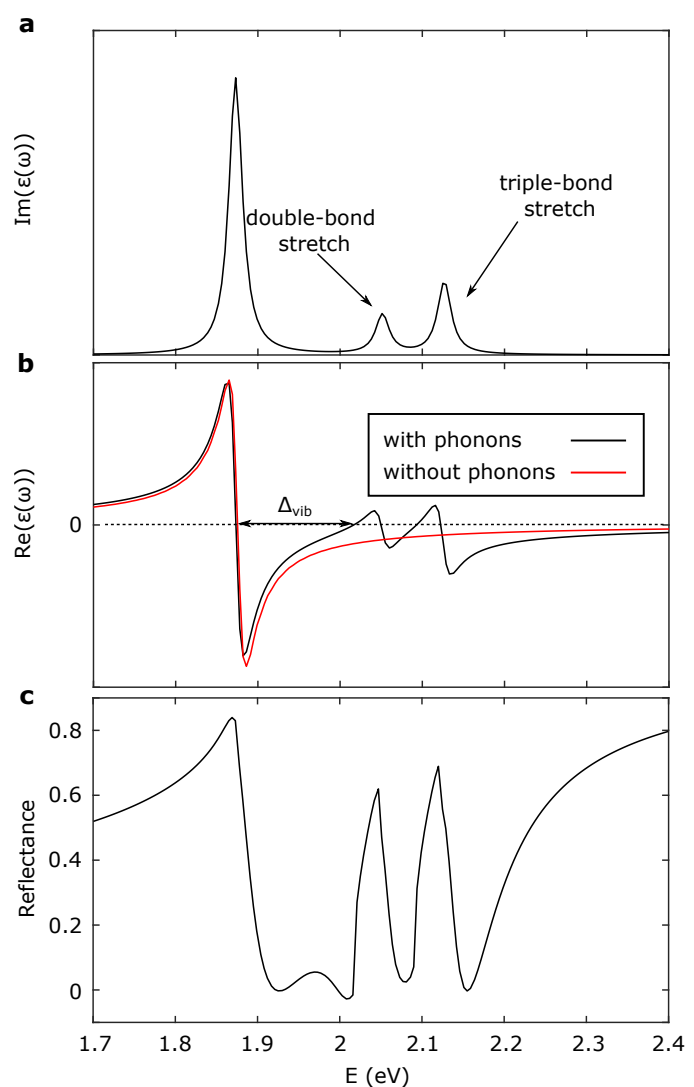

Figure 11 The imaginary (panel a) and real part (panel b) of the dielectric response of the model blue chains, once the effect of phonons is accounted for. The main extra contributions to the structure of $\epsilon(\omega)$, compared to the disorder-free case, arise due to the effect of the carbon-carbon double- and triple-bond stretch. Comparing the structure of $\operatorname{Re}(\epsilon(\omega))$ for the cases with and without phonons, we find that dynamic disorder leads to 'breaks' within the region of $\operatorname{Re}(\epsilon(\omega))<0$. This leads to a richer structure for the reflectance in panel c, compared to the disorder-free case of Figure 7

material closer to the metallic limit ${ }^{[58}$ It is hence not surprising that these motions have a large effect on the optical properties of the studied PDAs. For the computed exciton coherence length of $N=15$, we obtain $\Delta_{\text {vib }}$ values of $137 \mathrm{meV}$ and $123 \mathrm{meV}$ for the model blue and the model red chains respectively. We compute the associated reflectivity spectra in Figure 11, where we find that vibronic peaks introduce 'gaps' within the high reflectance region of the stop-band.

Therefore, accounting for vibrational effects allows one to predict part of the internal structure of the stopband of an exciton that is strongly coupled to a photon. However, the precise form of the reflectivity, depends very sensitively on the exciton coherence length $N$, as shown in Figure 12 , where we use the width $\Delta_{\text {vib }}$ as a proxy for the size of phonon-induced gaps in the reflectivity. This dependence of $\Delta_{\text {vib }}$ on $N$ enters through the Huang-Rhys factors of the extended system, see equation20. In turn, the exciton coherence length depends on the exciton group velocity and the period of phonon modes, as seen in equation 21 . Hence the interplay of phonons with excitons is established to be crucial for the interaction of the latter with light. 


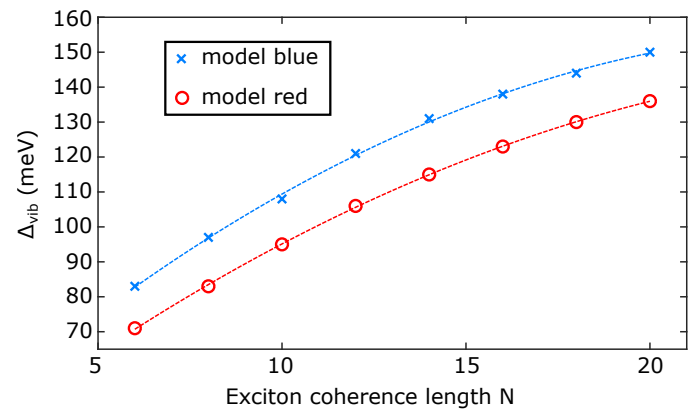

Figure 12 The dependence of the width $\Delta_{\text {vib }}$ of the two model PDA chains, on the exciton coherence length.

\section{Conclusions}

In this work, we have presented a first principles approach for obtaining the properties of exciton-polaritons in organic materials. Our theory requires input from a calculation of the material's dielectric response, and is agnostic to the methodology used to obtain it. By employing $G W$-BSE calculations for the electronic structure of polydiacetylenes, we obtain their stop-band width, which characterizes the strength of light-matter interactions. The results are in reasonable agreement with experiment, and we attribute the differences to our treatment of the materials as perfectly periodic, neglecting effects of disorder and additional excitons that weakly couple to light. We compute the speed of propagation of a polariton close to the resonance of light with an exciton, showing that this is an order of magnitude faster than purely excitonic motion. We find the conjugation length of polymer chains to be a critical parameter towards determining the magnitude of light-matter interactions, highlighting one manifestation of how static disorder can lead to weaker exciton-photon coupling. This effect is not important for the polydiacetylenes studied here, due to the purity of these materials. It could however be of relevance for different systems. Furthermore, we discuss the important role of dynamic disorder and show that vibrational effects introduce gaps within the frequency range of large reflectance that characterizes polaritons. We also discuss the screening of the Coulomb interaction provided by the side-chains of polydiacetylene as another potentially important contribution towards determining the magnitude of light-matter interactions. Thus all of the aforementioned factors appearing in realistic materials are important towards an accurate description of exciton-photon coupling. In principle, one needs to account for the above factors at the same time and self-consistently, something which is computationally challenging and will be the subject of a future work.

\section{Acknowledgments}

The authors acknowledge useful discussions with Richard Phillips (Cavendish) and Girish Lakhwani (University of Sydney). We thank Gianni Jacucci and Silvia Vignolini (Cambridge) for assistance with reflectivity measurements. We acknowledge the support of the Winton Programme for the Physics of Sustainability. A.M.A. acknowledges the support of the Engineering and Physical Sciences Research Council (EPSRC) for funding under grant EP/L015552/1. A.W.C. acknowledges financial support from Agence Nationale de la Recherche (Grant N. ANR-19-CE24-0028). The work in Mons is supported by the Belgian National Fund for Scientific 
Research (FRS-FNRS). Computational resources were provided by the Consortium des Équipements de Calcul Intensif (CECI). DB is FNRS Research Director.

\section{Data availability}

The data underlying this publication can be found in [URL added in proof].

\section{Appendix}

\section{A: Experimental reflectivity measurements}

Microscopic transmission and reflectance measurements were performed using a Zeiss AxioScope optical microscope in Köhler illumination equipped with a 100× objective (Zeiss EC Epiplan-APOCHROMAT 0.95 HD DIC) coupled to a spectrometer (Avantes HS2048) via an optical fibre (Thorlabs, FC-UV50-2-SR). Five spectra were collected for each sample using an integration time of $10 \mathrm{~ms}$ and $20 \mathrm{~ms}$ for reflection and transmission measurements, respectively. The reflectance and transmittance were calculated using a silver mirror (ThorLabs, PF10-03-P01) and the glass substrate as references respectively.

\section{B: Computational details}

\section{Electronic structure calculations}

We perform DFT calculations using the Quantum Espresso software package, 59 with the PBE functional together with a 60 Ry plane wave cutoff energy. For the model blue and model red chains, we employ $8 \times 4 \times 2$ and $4 \times 4 \times 2$ k-point grids respectively, while for the real red chains, we use $4 \times 4 \times 2$. For the model PDAs, we introduce a vacuum of $10 \AA$ between neighboring polymer chains, in order to minimize inter-chain interactions.

We perform energy self-consistent $G W$ calculations (i.e. $G W_{0}$ ) using the YAMBO code ${ }^{60}$ For the real chains, we find that including $400 \mathrm{Kohn}$-Sham states, 300 bands for the calculation of the polarization function, and a 7 Ry cutoff for the dielectric matrix, leads to converged values for the quasiparticle bandgaps. For the model blue (red) chains, we use 40 (80) Kohn-Sham states, 40 (80) bands for the calculation of the polarization function, and a 3 Ry cutoff for the dielectric matrix.

We solve the Bethe-Salpeter equation for the model blue (red) PDA using 4 (8) occupied and 4 (8) unoccupied bands, converging the position of the first exciton peak. For the exchange term in the Bethe-Salpeter kernel, the cutoff is set to 60 Ry. For the real red chains, we use 10 occupied and 10 unoccupied bands, and set the exchange to 40 Ry. In order to better emulate the experimental conditions, the incident electric field is averaged among the three spatial directions. The imaginary part of $\epsilon(\omega)$ is obtained by fitting a Lorentzian of $10 \mathrm{meV}$ width to the computed excitonic eigenvalues of the BSE Hamiltonian. The broadening of the absorption spectrum due to vibrational effects as presented in Figure 11 is then added on top of that, along with the additional peaks. 
Due to the one-dimensional character of the model PDAs, divergences can occur at small values of $q$ (i.e. at long distances) in the Coulomb term that appears in the many-body calculations. In order to avoid this problem, we use the random integration method, as implemented in YAMBO. We converge the calculations using $10^{5}$ random q-points in the first Brillouin zone, and by setting the cutoff for the real space components of the Coulomb interaction to $20 \mathrm{Ry}$.

For the model PDAs, we estimate the dispersion of the exciton band along $\Gamma-X$ as follows. We generate a supercell of size $2 \times 1 \times 1$, thus halving the size of the first Brillouin zone. As a result, the zone-boundary $X$ point folds onto $\Gamma$, which appears as an extra exciton state once the BSE Hamiltonian of the $2 \times 1 \times 1$ system is diagonalized. The distance of this new band from the exciton value at $\Gamma$ gives the width of the exciton band, which we find to be equal to $1.33 \mathrm{eV}$.

All the aforementioned electronic structure calculations are performed on the PDA structures as obtained from X-ray diffraction (for the real red system), or on the structures as obtained from X-ray diffraction and following the substitution of the side-chains with hydrogen atoms. A geometry optimization of these structures is not carried out, as the bond-length alternation of these one-dimensional carbon chains is known to be extremely sensitive to the fraction of exact exchange that the employed DFT functional includes 61 It has been shown that while hybrid functionals perform better than GGA, $\underset{61}{10}$ it necessary to use very accurate methods such as Quantum Monte Carlo in order to obtain geometries with realistic bond-length alternations, which is beyond the scope of this work. As discussed in the main manuscript, the exciton energy is extremely sensitive to the precise value of the bond-length alternation. Therefore we limit ourselves to obtaining exciton energies for unoptimized structures, and only optimize the geometry of the studied structures prior to phonon calculations,

\section{Phonon calculations}

We calculate the lattice dynamics of the different PDA variants in the harmonic approximation using finite differences $39 \sqrt[30]{6}$ We calculate the dynamical matrix on a $1 \times 1 \times 1$ coarse q-point grid. Therefore, we only account for the effect of $\Gamma$-point phonons, which provides a good approximation, since these are most strongly coupled to the zero-momentum exciton states that result from photoexcitation. Prior to phonon calculations, the geometry of the different PDAs is optimized using the PBE functional.

\section{Dependence of polariton splitting on peak width}

Figure 13 visualizes the value of $\Delta_{\text {vib }}$ of the model blue PDA, within a range of values for the width of the exciton and vibronic peaks, ranging from $5 \mathrm{meV}$ to $50 \mathrm{meV}$. We find that $\Delta_{\text {vib }}$ does not vary by more than $10 \mathrm{meV}$ within this range of widths. Here an exciton coherence length $N=15$ has been used. 


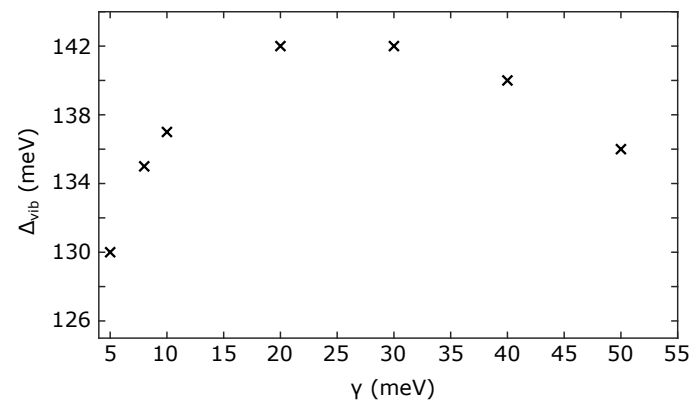

Figure 13 The dependence of the width $\Delta_{\text {vib }}$ of the first region with $\operatorname{Re}(\epsilon(\omega))$ on the width $\gamma$ of the exciton and vibronic peaks.

\section{References}

${ }^{1}$ S. Pekar. The Theory of Electromagnetic Waves in a Crystal in which Excitons Are Produced. Soviet Journal of Experimental and Theoretical Physics, 6(4):785, 1958.

${ }^{2}$ S. I. Pekar. Identification of excitons with light waves in a crystal. Jetp, 11(6):1286, 1960.

${ }^{3}$ J. J. Hopfield. Theory of the contribution of excitons to the complex dielectric constant of crystals. Physical Review, 112(5):1555-1567, 1958.

${ }^{4}$ Heinz Kalt and Claus F Klingshirn. Semiconductor Optics 1. Springer, Fifth edition, 2019. Chapter 8.

${ }^{5}$ Sebastian Reineke, Frank Lindner, Gregor Schwartz, Nico Seidler, Karsten Walzer, Björn Lüssem, and Karl Leo. White organic light-emitting diodes with fluorescent tube efficiency. Nature, 459(7244):234-8, 2009.

${ }^{6}$ Lingxian Meng, Yamin Zhang, Xiangjian Wan, Chenxi Li, Xin Zhang, Yanbo Wang, Xin Ke, Zuo Xiao, Liming Ding, Ruoxi Xia, Hin-Lap Yip, Yong Cao, and Yongsheng Chen. Organic and solution-processed tandem solar cells with 17.3\% efficiency. Science, 361(6407):1094-1098, 2018.

${ }^{7}$ Artem Strashko, Peter Kirton, and Jonathan Keeling. Organic Polariton Lasing and the Weak to Strong Coupling Crossover. Physical Review Letters, 121(19):193601, 2018.

${ }^{8}$ S. Kéna-Cohen and S. R. Forrest. Room-temperature polariton lasing in an organic single-crystal microcavity. Nature Photonics, 4(6):371-375, 2010.

${ }^{9}$ M. Knupfer. Exciton binding energies in organic semiconductors. Applied Physics A, 77(5):623-626, Oct 2003.

${ }^{10}$ A. Köhler and H. Bässler. Triplet states in organic semiconductors. Materials Science and Engineering R: Reports, 66(4-6):71-109, 2009.

${ }^{11}$ D. Beljonne, H. Yamagata, J. L. Brédas, F. C. Spano, and Y. Olivier. Charge-transfer excitations steer the davydov splitting and mediate singlet exciton fission in pentacene. Physical Review Letters, 110(22):1-5, 2013. 
12 Timothy C. Berkelbach, Mark S. Hybertsen, and David R. Reichman. Microscopic theory of singlet exciton fission. II. Application to pentacene dimers and the role of superexchange. Journal of Chemical Physics, 138(11), 2013.

${ }^{13}$ Shane R Yost, Jiye Lee, Mark W B Wilson, Tony Wu, David P McMahon, Rebecca R Parkhurst, Nicholas J Thompson, Daniel N Congreve, Akshay Rao, Kerr Johnson, Matthew Y Sfeir, Moungi G Bawendi, Timothy M Swager, Richard H Friend, Marc a Baldo, and Troy Van Voorhis. A transferable model for singlet-fission kinetics. Nature Chemistry, 6(6):492-497, 2014.

${ }^{14}$ Pedro B. Coto, Sahar Sharifzadeh, Jeffrey B. Neaton, and Michael Thoss. Low-lying electronic excited states of pentacene oligomers: A comparative electronic structure study in the context of singlet fission. Journal of Chemical Theory and Computation, 11(1):147-156, 2015.

${ }^{15}$ M. Litinskaya, P. Reineker, and V. M. Agranovich. Exciton-polaritons in organic microcavities. Journal of Luminescence, 119-120(SPEC. ISS.):277-282, 2006.

${ }^{16}$ D. G. Lidzey, D. D.C. Bradley, M. S. Skolnick, T. Virgili, S. Walker, and D. M. Whittaker. Strong excitonphoton coupling in an organic semiconductor microcavity. Nature, 395(6697):53-55, 1998.

${ }^{17}$ Daniel Polak, Rahul Jayaprakash, Thomas P. Lyons, Luis Martínez-Martínez, Anastasia Leventis, Kealan J. Fallon, Harriet Coulthard, David G. Bossanyi, Kyriacos Georgiou, Anthony J. Petty, John Anthony, Hugo Bronstein, Joel Yuen-Zhou, Alexander I. Tartakovskii, Jenny Clark, and Andrew J. Musser. Manipulating molecules with strong coupling: Harvesting triplet excitons in organic exciton microcavities. Chemical Science, 11(2):343-354, 2020.

${ }^{18}$ V. M. Agranovich, M. Litinskaia, and D. G. Lidzey. Cavity polaritons in microcavities containing disordered organic semiconductors. Physical Review B - Condensed Matter and Materials Physics, 67(8):1-10, 2003.

${ }^{19}$ Alessandro Troisi, Giorgio Orlandi, and John E. Anthony. Electronic interactions and thermal disorder in molecular crystals containing cofacial pentacene units. Chemistry of Materials, 17(20):5024-5031, 2005.

${ }^{20}$ Alessandro Troisi and Giorgio Orlandi. Dynamics of the intermolecular transfer integral in crystalline organic semiconductors. Journal of Physical Chemistry A, 110(11):4065-4070, 2006.

${ }^{21}$ Pierluigi Cudazzo, Francesco Sottile, Angel Rubio, and Matteo Gatti. Exciton dispersion in molecular solids. Journal of Physics Condensed Matter, 27(11), 2015.

${ }^{22}$ Sivan Refaely-Abramson, Felipe H. Da Jornada, Steven G. Louie, and Jeffrey B. Neaton. Origins of Singlet Fission in Solid Pentacene from an ab initio Green's Function Approach. Physical Review Letters, 119(26):1-6, 2017.

${ }^{23}$ Antonios M. Alvertis, Raj Pandya, Loreta A. Muscarella, Nipun Sawhney, Malgorzata Nguyen, Bruno Ehrler, Akshay Rao, Richard H. Friend, Alex W. Chin, and Bartomeu Monserrat. The impact of exciton delocalization on exciton-vibration interactions in organic semiconductors. 2020. arXiv:2006.03604. 
${ }^{24}$ R. F. Mahrt and H. Bässler. Light and heavy excitonic polarons in conjugated polymers. Synthetic Metals, 45(1):107-117, 1991.

${ }^{25}$ Frank C. Spano. The spectral signatures of frenkel polarons in H- And J-aggregates. Accounts of Chemical Research, 43(3):429-439, 2010.

${ }^{26}$ H. Yamagata and F. C. Spano. Vibronic coupling in quantum wires: Applications to polydiacetylene. Journal of Chemical Physics, 135(5), 2011.

${ }^{27}$ N. Somaschi, L. Mouchliadis, D. Coles, I. E. Perakis, D. G. Lidzey, P. G. Lagoudakis, and P. G. Savvidis. Ultrafast polariton population build-up mediated by molecular phonons in organic microcavities. Applied Physics Letters, 99(14), 2011.

${ }^{28}$ Ning Wu, Johannes Feist, and Francisco J. Garcia-Vidal. When polarons meet polaritons: Exciton-vibration interactions in organic molecules strongly coupled to confined light fields. Physical Review B, 94(19), 2016.

${ }^{29}$ David M. Coles, Paolo Michetti, Caspar Clark, Wing Chung Tsoi, Ali M. Adawi, Ji Seon Kim, and David G. Lidzey. Vibrationally assisted polariton-relaxation processes in strongly coupled organic-semiconductor microcavities. Advanced Functional Materials, 21(19):3691-3696, 2011.

30 Javier Del Pino, Florian A.Y.N. Schröder, Alex W. Chin, Johannes Feist, and Francisco J. Garcia-Vidal. Tensor network simulation of polaron-polaritons in organic microcavities. Physical Review B, 98(16):165416, 2018.

31 Javier Del Pino, Florian A. Y. N. Schröder, Alex W. Chin, Johannes Feist, and Francisco J. Garcia-Vidal. Tensor network simulation of non-Markovian dynamics in organic polaritons. Physical Review Letters, $121(22): 227401,2018$.

${ }^{32}$ Harmut Haug and Stephan W. Koch. Quantum Theory of the Optical and Electronic Properties of Semiconductors. World Scientific, Fourth edition, 2004. Chapter 11.

${ }^{33}$ Florian A. Y. N. Schröder, David H. P. Turban, Andrew J. Musser, Nicholas D. M. Hine, and Alex W. Chin. Tensor network simulation of multi-environmental open quantum dynamics via machine learning and entanglement renormalisation. Nature Communications, 10(1):1062, 2019.

${ }^{34}$ Antonios M. Alvertis, Florian A.Y.N. Schröder, and Alex W. Chin. Non-equilibrium relaxation of hot states in organic semiconductors: Impact of mode-selective excitation on charge transfer. Journal of Chemical Physics, $151(8), 2019$.

${ }^{35}$ Hedin Lars. New Method for Calculating the One-Particle Green's Function with Application to the ElectronGas Problem. Physical Review, 139(17):796-823, 1965.

${ }^{36}$ Michael Rohlfing and Steven G. Louie. Electron-hole excitations in semiconductors and insulators. Physical Review Letters, 81(11):2312-2315, 1998. 
${ }^{37}$ Michael Rohlfing and Steven G. Louie. Electron-hole excitations and optical spectra from first principles. Physical Review B - Condensed Matter and Materials Physics, 62(8):4927-4944, 2000.

${ }^{38}$ Michael Rohlfing and Steven G Louie. Optical Excitations in Conjugated Polymers. Physical Review Letters, 82(9):1-4, 1999.

${ }^{39}$ K. Kunc and Richard M. Martin. Ab initio force constants of GaAs: A new approach to calculation of phonons and dielectric properties. Physical Review Letters, 48(6):406-409, 1982.

${ }^{40}$ Bartomeu Monserrat. Electron - phonon coupling from finite differences. Journal of Physics Condensed Matter, 30, 2018.

${ }^{41}$ John David Jackson. Classical electrodynamics. Wiley, New York ; London, 2nd ed. edition, 1975.

${ }^{42}$ S. Dai, Z. Fei, Q. Ma, A. S. Rodin, M. Wagner, A. S. McLeod, M. K. Liu, W. Gannett, W. Regan, K. Watanabe, T. Taniguchi, M. Thiemens, G. Dominguez, A. H. Castro Neto, A. Zettl, F. Keilmann, P. Jarillo-Herrero, M. M. Fogler, and D. N. Basov. Tunable phonon polaritons in atomically thin van der waals crystals of boron nitride. Science, 343(6175):1125-1129, 2014.

${ }^{43}$ Anatoly V. Zayats, Igor I. Smolyaninov, and Alexei A. Maradudin. Nano-optics of surface plasmon polaritons. Physics Reports, 408(3-4):131-314, 2005.

${ }^{44}$ J. Lagois and B. Fischer. Chapter 2 - Surface Exciton Polaritons from an Experimental Viewpoint. In D.L. Mills. V.M. Agranovich, editor, Surface polaritons, Modern problems in condensed matter sciences. North-Holland Pub. Co. : Sole distributors for the USA and Canada, Elsevier Science Pub. Co., Amsterdam ; New York, 1982.

${ }^{45}$ M. Schott and G. Wegner. Chapter III-1 - Basic Structural and Electronic Properties of Polydiacetylenes. In D.S. Chemla and J. Zyss, editors, Nonlinear Optical Properties of Organic Molecules and Crystals, pages 3 49. Academic Press, 1987.

${ }^{46}$ P A R Pierre Spinat, Catherine Brouty, Annick Whuler, and E T Marie-claude Sichi. Determination Structurale a $205 \mathrm{~K}$ du Poly \{ bis [( p-bromophenylcarbamoyloxy ) -4 butyl ] - 1, 2 butene-1 yne-3 ylene \}, ( $\mathrm{C}_{26} \mathrm{H}_{26} \mathrm{Br}_{2} \mathrm{~N}_{204}$ ) n. Acta Crystallographica, 41:1452-1455, 1985.

${ }^{47}$ V. Enkelmann and G. Schleier. Poly[1,2-bis(diphenylaminomethyl)-1-buten-3-ynylene]. Acta Crystallographica Section B Structural Crystallography and Crystal Chemistry, 36(8):1954-1956, 1980.

${ }^{48}$ T. Barisien, L. Legrand, G. Weiser, J. Deschamps, M. Balog, B. Boury, S. G. Dutremez, and M. Schott. Exciton spectroscopy of red polydiacetylene chains in single crystals. Chemical Physics Letters, 444(4-6):309$313,2007$.

${ }^{49}$ Michel Schott. The colors of polydiacetylenes: A commentary. Journal of Physical Chemistry B, 110(32):15864-15868, 2006. 
${ }^{50}$ Michael R. Philpott. Optical Reflection Spectroscopy of Organic Solids. Annu Rev. Phys. Chem., 31:97-129, 1980.

${ }^{51}$ Stefan C.J. Meskers and Girish Lakhwani. Reflection of light by anisotropic molecular crystals including exciton-polaritons and spatial dispersion. Journal of Chemical Physics, 145(19), 2016.

${ }^{52}$ M. Tristani-Kendra, C.J. Eckhardt, J. Bernstein, and E. Goldstein. Strong coupling in the optical spectra of polymorphs of a squarylium dye. Chemical Physics Letters, 98(1):57-61, 1983.

${ }^{53}$ Jean Sébastien Filhol, Jérôme Deschamps, Sylvain G. Dutremez, Bruno Boury, Thierry Barisien, Laurent Legrand, and Michel Schott. Polymorphs and colors of polydiacetylenes: A first principles study. Journal of the American Chemical Society, 131(20):6976-6988, 2009.

${ }^{54}$ A. Horvath, G. Weiser, C. Lapersonne-Meyer, M. Schott, and S. Spagnoli. Wannier excitons and FranzKeldysh effect of polydiacetylene chains diluted in their single crystal monomer matrix. Physical Review B Condensed Matter and Materials Physics, 53(20):13507-13514, 1996.

${ }^{55}$ Fred Wudl and Steven P Bitler. Synthesis and Some Properties of Poly(diacetylene) (Polyenyne) Oligomers. Journal of the American Chemical Society, 108(15):4685-4687, 1986.

${ }^{56}$ Frank C. Spano. Absorption and emission in oligo-phenylene vinylene nanoaggregates: The role of disorder and structural defects. Journal of Chemical Physics, 116(13):5877-5891, 2002.

${ }^{57}$ F. C. Spano. Optical microcavities enhance the exciton coherence length and eliminate vibronic coupling in J-aggregates. Journal of Chemical Physics, 142(18), 2015.

${ }^{58}$ S. Kivelson and A. J. Heeger. First-Order Transition to a Metallic State in Polyacetylene: A Strong-Coupling Polaronic Metal. Physical Review Letters, 55(3):308-311, 1985.

${ }^{59}$ Paolo Giannozzi, Stefano Baroni, Nicola Bonini, Matteo Calandra, Roberto Car, Carlo Cavazzoni, Davide Ceresoli, Guido L. Chiarotti, Matteo Cococcioni, Ismaila Dabo, Andrea Dal Corso, Stefano De Gironcoli, Stefano Fabris, Guido Fratesi, Ralph Gebauer, Uwe Gerstmann, Christos Gougoussis, Anton Kokalj, Michele Lazzeri, Layla Martin-Samos, Nicola Marzari, Francesco Mauri, Riccardo Mazzarello, Stefano Paolini, Alfredo Pasquarello, Lorenzo Paulatto, Carlo Sbraccia, Sandro Scandolo, Gabriele Sclauzero, Ari P. Seitsonen, Alexander Smogunov, Paolo Umari, and Renata M. Wentzcovitch. QUANTUM ESPRESSO: A modular and open-source software project for quantum simulations of materials. Journal of Physics Condensed Matter, 21(39), 2009.

${ }^{60}$ Andrea Marini, Conor Hogan, Myrta Grüning, and Daniele Varsano. yambo: An ab initio tool for excited state calculations. Computer Physics Communications, 180(8):1392-1403, 2009.

${ }^{61}$ E. Mostaani, B. Monserrat, N. D. Drummond, and C. J. Lambert. Quasiparticle and excitonic gaps of one-dimensional carbon chains. Physical Chemistry Chemical Physics, 18(22):14810-14821, 2016. 